\title{
The study on the spectrum sensing technology in wireless cognitive network
}

\author{
Bing $\mathrm{Hu}$ \\ Shenyang polytechnic college, Shenyang, China \\ a2238552865@qq.com
}

\begin{abstract}
Keywords: Cognitive wireless network; Spectrum perception; Matched filtering; Smooth circulation
\end{abstract} characteristic; Energy detection

\begin{abstract}
As a kind of technology with an application prospect, cognitive network not only can improve the utilization of spectrum resource, but also can relieve the increasingly serious problems of spectrum resources tension fundamentally, and it has become a research focus in the field of wireless communications, and gets more and more attention. This technology improve the utilization rate of spectrum by allowing cognitive users free access to the authorized spectrum, to ensure the spectrum resources can be recycled. In a number of key technologies of cognitive radio, spectrum detection algorithm occupies a very important position, it needs to detect the utilization of spectrum resource in real-time and accurately. This article introduces the concept and development present situation of cognitive network first, elaborated key technology of cognitive network, and made intensive study on the key technology of spectrum perception and the existing problems.
\end{abstract}

\section{Introduction}

The spectrum that communication system allocated became extremely crowded with the rapid development of information and communication technology, and the interference phenomenon is serious, all of this have restrict the continue development of Wireless Sensor Network(WSN).In order to solve the problem of limited band resources, we can introduce cognitive radio technology into sensor network, set up suitable spectrum using model, so as to improve the efficiency of the use of the frequency band.[1,2,3]As a new generation wireless communication technology, cognitive radio technology is mainly used in the radio and television band which is the recent research of IEEE802.22 working team, and wireless cognitive network technology will have a broad application prospect with the continuous development of wireless communication technology. The technology can homogeneous learning, changing some operating parameters real-time with purpose, so as to solve the problem of increasingly shortage of spectrum resources. Improve the utilization rate of spectrum by allowing cognitive users free access to the authorized spectrum, to ensure the spectrum resources can be recycled. At present, the cognitive wireless network has become a hot research topic in the field of communications. Its core idea is that wireless communication equipment can perceive the usage of spectrum resources dynamically, get access to free spectrum dynamically, and don't disturb the normal work of the authorized user at the same time[4]. With the introduction of cognitive wireless networks, the network node can perception spectrum information in the surrounding environment real-timely, obtain available spectrum resources, and it can also select non-hold spectrum resources in the process of communication dynamically to realize the communication process. This paper introduces the concept, current situation of the development of cognitive wireless network, analysis of the key technology of cognitive network and frequency spectrum, and the present problems of perception. The main content of this article includes:

a. The concept and the current situation of the development of cognitive wireless network

b. Cognitive network technical analysis

c. Key technologies of spectrum perception and existing problems

\section{The Concept and Current Development Situation of Cognitive Network}

The concept of Cognitive Radio(CR) originated from Dr Joseph Mitola's lifetime work in 1999, Its 
core idea is that $\mathrm{CR}$ has the ability to learn, it can interact information with the surrounding environment, to perceive and use the available spectrum in the space, and limit and reduce the happening chance of the conflict [5].It is generally believed that the cognitive network is that which can perceive the external environment, adjust the internal configuration of communication network, adapt to changes in the external environment intelligently through the understanding and study on the external environment. It is generally believed that the cognitive network is through the understanding and study of the external environment, perceive the external environment, adjust the internal configuration of communication network, adapt to changes in the external environment intelligently. Its main purpose is to provide customers with the most optimal end-to-end performance. Cognitive radio technology can solve the contradiction between the spectrum demand and the limited spectrum resources effectively, detect the idle spectrum resource, and implement spectrum sharing in the case of no interference with authorized users, solved the problem of low utilization of frequency spectrum effectively. Spectrum sensing is one of the key technology of the physical cognitive radio, the cognitive radio spectrum which has pass the examining can avoid interference and improve spectrum efficiency. Spectrum sensing technology include three types: detection based on the transmitter, collaborative detection and detection based on the interference. In the cognition of radio network, there are many types of authorized user signal and diversity of wireless channel transmission characteristics, and authorized user system and the interference level it can withstand, high demands are put forward on the performance of spectrum detection, in order to make interference to the authorized users to a minimum, cognitive radio users must release the spectrum quickly when authorized user takes up the spectrum, this requires a spectrum sensing algorithm with characteristics of short perception time, low complexity, high timeliness, these have increased the difficulty of spectrum sensing technology greatly. Therefore, there is a great significance to research cognitive radio spectrum sensing technology.

It attracted wide attention in the industry when the cognitive radio is proposed. Representative (DARPA), the next generation wireless communication (XG) project which be funded has achieved some success, Its main research is system methods and key technologies, so as to realize dynamic spectrum application which is based on cognitive radio technology, since 2003, Raytheon company signed the agency contract of the next generation wireless communication plan with the United States defense advanced research projects, they engaged in the research and development of cognitive radio technology mainly; Genia wireless communications technology research center mainly study the genetic algorithm and the cognitive radio nodes based on the cognition, the research of mobile communication technology in Britain is turning to cognitive radio, the multi-mode terminals team and communication systems research center at the university of Bristol began to research the adaptive radio-frequency technique together; the projects such as DRiVE, Over DRiVE and the TRUST funded by European communication association study spectrum dynamic allocation and flow control in the mix, radio network mainly, in the meantime, the project of end to end also be funded by association, and it mainly studies how to integrate different types of wireless network in the future by using the end to end reconfiguration of the network and software radio technology, and have made some study on the market model based on the application of cognitive radio, pricing strategy of cognitive radio network and accounting strategy; at the university of California, Berkeley, set up the simulation platform, make simulation and performance analysis on the various spectrum audience experiment. Adaptive spectrum radio (adaptive spectrum radio, ASR) test platform developed by MITRE company demonstrates the feasibility of adaptive spectrum successfully. In addition, the delft university Dutch, the Berlin institute of technology, also made study on cognitive radio. In the field of civil, MOTOROLA, Intel and other companies are also set up their research group, and began to carry out the related research on cognitive radio.

\section{The Spectrum Sensing Technology}

Spectrum sensing technology is one of the main core technology of cognitive network. Its purpose is to discover spectrum holes in the time domain, frequency and spatial domain, provide cognitive 
users with the opportunity to use spectrum.

Spectrum sensing technology include the following three parts, they are detection based on interference, primary user signal detection and collaborative detection. The spectrum sensing technology is mainly based on the main user transmitter detection, it is shown in Fig. 1.

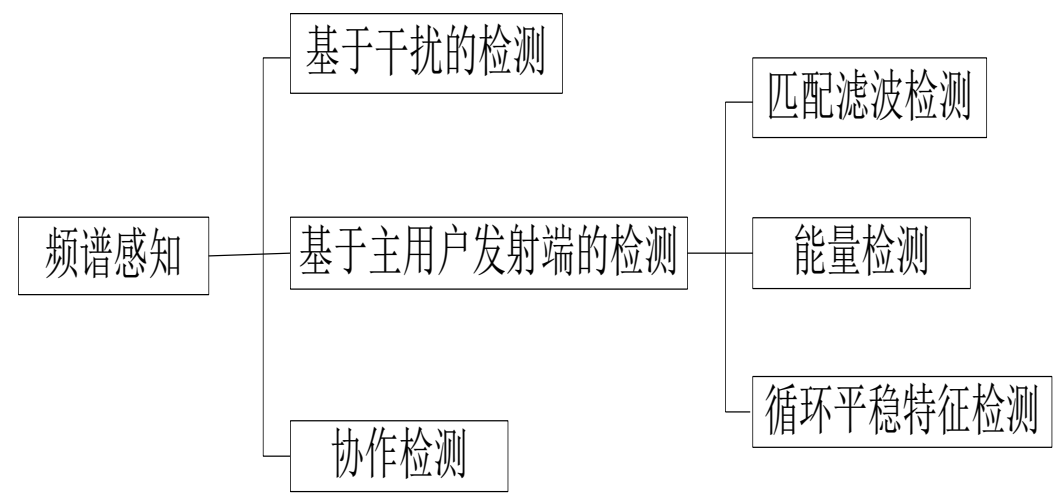

Figure 1. Classification of spectrum perception technology

Matched Filter Detection. If the primary user signal is known deterministic signal, matched filter is the optimum detector under the condition of Additive White Gaussian Noise (AWGN), it can maximize the output signal-to-noise ratio, matched filter detection belongs to the coherent detection of signals, detection statistics is:

$$
T(x)=\sum_{n=1}^{N} x(n) \stackrel{*}{*}(n)
$$

Decision criteria as follows:

$$
H_{0}: T(x) \prec \lambda, H_{1}: T(x) \succ \lambda
$$

Compared the decision statistic with predetermined threshold value $\lambda$,if it is greater than the threshold value $\lambda$,it said that there are authorized users are using the frequency band; If less than the threshold value $\lambda$,we can know there is only the noise in the frequency band, authorized users didn't use the frequency band, cognitive users can use the frequency band at this time.

The advantages of matched filter detection: can testing the main user exist whether or not fast and accurately, disadvantages: it needs to know the authorized users information in advance, there would be special receivers to authorized users, need to synchronous with frequency periodically. In addition, the amount of calculation is also bigger, if the prior knowledge is not accurate, the performance of matched filter will be reduced dramatically.

Cyclic Stationary Feature Detection. Wireless communication signal has the character of cyclic stability, and noise and interference are stationary process commonly, don't have this cycle stability Don't have this cycle stability, so we can detect whether the primary user signal appeared through the analysis of the frequency spectrum self-correlation function of signal in the process of the spectrum sensing of cognitive network.

Assumes that the channel is the ideal, its model is:

$$
y(t)=x(t)+n(t)
$$

In the formula, $\mathrm{n}(\mathrm{t})$ is the Additive White Gaussian Noise which mean is zero and variance is $\sigma^{2}$, $y(t)$ is the receiving signals.

Assume the spectral correlation function of signal and noise respectively are $S_{x}^{\alpha}(f)$, $S_{w}^{\alpha}(f)$ signal and noise are uncorrelated, so the spectral correlation function of the received signal y 
(t) is as follows:

$$
S_{y}^{\alpha}(f)=S_{x}^{\alpha}(f)+S_{w}^{\alpha}(f)
$$

$w(t)$ did not present spectrum related features on the second-order cyclic frequency because it is the Gaussian noise, there is:

$$
S_{w}^{\alpha}(f)=0, \alpha \neq 0
$$

So we can detect the signal of authorized users use the following signal detection rules, when $\alpha \neq 0$,

$$
S_{y}^{\alpha}(f)=\left\{\begin{array}{cc}
S_{x}^{\alpha}(f) & H_{1}, \\
0 & H_{0} .
\end{array}\right.
$$

The advantages of cycle stationary feature detection: it can distinguish the noise energy from the modulation signal power, the premise is that the noise should be irrelevant generalized stationary signal. Therefore, detection of cycle stationary process characteristics can be tested under low SNR signals. The disadvantage: high computational complexity.

Literature[6] researched the spectrum detection problem in low SNR environment according to the features of signal cycle stable spectrum, and put forward a kind of adaptive decision threshold spectrum detection algorithm based on cyclic spectrum energy.

Energy Detection. Energy detection is the simplest and most classic signal detection method, and it is also the hot issue of current research. Assuming that authorized user signal $\mathrm{s}(\mathrm{n})$ and noise signal $\mathrm{w}(\mathrm{n})$ both are Gaussian distribution, the mean and variance are $\mu_{1}, \mu_{2}$ and $\sigma_{1}, \sigma_{2}$ respectively. There are two cases when detect, one kind is that authorized users are taking up the spectrum, they received the signal which include authorized user signal and noise signal, another case is the authorized user did not take up of the spectrum, the received signal contains noise signal only, as shown in the formula (7):

$$
H 1: y(n)=s(n)+w(n)
$$

HO: $y(n)=w(n)$.

Energy detector carry on $\mathrm{N}$ point sampling to received signal at first, and then time domain detection calculating absolute value and the sum of squares on the sampling values directly, frequency domain detection do Fast Fourier Transform, Fast Fourier Transform and FFT)on the sampling point at first, switch to the frequency domain, then make the sum of squares computing based on modulus value of the frequency domain point, get the energy value $\mathrm{E}$ of received signal, as shown in the formula(8):

$$
E=\sum_{n=1}^{N}\left|y_{n}\right|^{2}
$$

In the formula, yn is the Nth sampling signal, $N=2 T W$ is the sampling size, and there $\mathrm{T}$, $\mathrm{W}$, is the sampling time and bandwidth respectively. Finally compared the energy calculated value we got with the threshold which we set up before, if it is bigger than the decision threshold, there is a signifying that the spectrum is taken up by authorized users. And the frequency domain detection can also distinguish the part which is occupied by authorized users.

The advantages of energy detection method: simple relatively, implement easily, in addition, it is a non-coherent detection, less demand on phase synchronization. The disadvantages: detection performance is poorer under the low SNR; vulnerable by interference; not suitable for the detection of direct sequence signal, spread spectrum signal and frequency hopping signal, and can't distinguish the type of authorized users.

Literature [7] lets secondary user transmitting terminal adjust the threshold based on 
instantaneous SINR (Signal to Interference plus Noise the wire) dynamically, its purpose is maximize the average of transmission rate and throughput of secondary users within the scope of the interference limit to primary user. The task of this paper is to determine an adaptive threshold control strategy function, and achieve the above purpose by calculation threshold, for example SINR function. Literature [8] proposed a cooperative energy detection scheme of CRSN, and tested detection probability of the proposed scheme in some typical fading channel environment. The simulation results show that its performance is no less than Neyman-Pearson detector in the case of calculation is lower than the other.

Cooperative Spectrum Sensing. Collaborative spectrum sensing is proposed mainly based on the problem of noise uncertainty, decline and the shadow in the spectrum perception, it can reduce the missed detection probability Pm and false alarm probability Pf. In addition, the collaborative perception can also solve the problem of hidden users, reduce the perception time. Mainly include centralized collaborative perception and distributed collaborative perception.

Centralized collaborative perception: collaboration center collect local observations from the secondary users, decide the available channel with the help of decision fusion rules and the information of the channel which users access to.

Distributed collaboration awareness: the secondary users can exchanged local decision results without the help of collaboration center, reduce the cost.

The problems of collaborative perception are as follows:

a. The secondary user cannot bear complex hardware equipment and complex calculations.

b. When the broadband collaborative perception, the secondary user must scanning frequency channel for large scale, share the test results, which leads to a vast energy consumption and the increase of invalid data throughput.

c. Under the environment of dynamic spectrum, the real-time performance of user's information collection is poor due to users movement and channel fading.

We make research on the users selected, decision fusion and centralized, distributed collaborative perception about collaborative spectrum sensing at present.

The literature [9] proposes a new collaborative spectrum sensing monitoring mechanism based on Stochastic Resonance (SR) technology and optimization of monitoring time slot. Simulation proves that, the detection performance of the new algorithm proposed in this paper has improved significantly than the traditional ED algorithm in the case of low SNR. Literature [10] introduces a new Double Eigenvalue value Threshold (DET) collaborative spectrum method which is based on the random matrix theory, testing the spectrum hole use simple covariance matrix characteristic value. The simulation results show that DET performance is superior to the Maximum Eigenvalue Detection (MED) and the Energy Detection (ED) under the condition of the same computational complexity.

\section{Challenges of Cognitive Network Wireless Spectrum Perception}

In recent years, people's research of cognitive wireless network have achieved fruitful results. But with the continuous development of wireless communication technology, there are still problems as the following aspects need to solve:

The spectrum sensing technology of wireless sensor network that suitable for low energy consumption, low computational complexity.

Hidden primary user problems is similar to the problem of hidden nodes in Carrier Sense Multiple Access (CSMA). There may be many causes for this problem, such as severe multi-path fading and the shadow effect detected by secondary user in scanning the main users launch. How to solve the problem of the hidden users will become the focus of the future research.

The optimized collaboration sensing. How to distribution the perception operation of CRSN quarter point concertedly, how to optimize collaboration perception results of CRSN node and the resulting network overhead, energy consumption, delay and other factors. 


\section{Conclusions}

Spectrum is a precious resource in wireless communication system, the spectrum has been the focus of the research in recent decades. Cognitive wireless network as a method of chance to access and use the spectrum more efficient has become the most potential fields. Detection of spectrum opportunity is the most important part of cognitive wireless network, namely the spectrum perception. This paper summarized some key problems of cognitive network, and analyze the existing research results from the aspects of spectrum sensing. This paper emphasize the research of spectrum sensing technology according to the theoretical and practical problems in the practical development process of cognitive network, and probes into the hot issue which needs to a further study.

\section{References}

[1] Devroye, N., Vu, M., and Tarokh, V., "Cognitive radio networks". IEEE Signal Processing Mag.,

[1] Nov. 2008, vol. 25, pp. 12-23.

[2] Yucek, T., and Arslan, H., "A survey of spectrum sensing algorithms for cognitive radio applications". IEEE Commun. Surveys Tutorials, quarter. 2009, vol. 11, no.1, pp. 116-130.

[3] Wang, B., and Liu, K. J. R. “Advances in cognitive radio networks: A survey”. IEEE J. Sel. Topics Signal Process, Feb. 2011, vol. 5, no. 1, pp. 5-23.

[4] Haykin, S., Thomson, D., and Reed, J. "Spectrum sensing for cognitive radio". Proc. IEEE, May 2009, vol. 97, no. 5, pp. 849-877.

[5] Ghasemi, A., and Sousa, E., "Spectrum sensing in cognitive radio networks: requirements, challenges and design trade-offs". IEEE Commun. Mag, Apr. 2008, vol. 46, no. 4, pp. 32-39.

[6] ZENG Y H, LIANG Y C. "Eigenvalue-based spectrum sensing algorithms for cognitive radio". IEEE Transactions on Communications, 2009, vol. 57, no. 6, pp.1784-1793.

[7] He, A., Bae, K. K., and Newman, T., "A survey of artificial intelligence for cognitive radios". IEEE Trans. Veh. Technol., May 2010, vol. 59, no. 4, pp. 1578-1592.

[8] Dong, X., Li, Y., and $\mathrm{Wu}, \mathrm{C}$. “A learner based on neural network for cognitive radio". in $12^{\text {th }}$ IEEE International Conference on Communication Technology (ICCT '10), Nanjing, China, Nov 2010, pp. 893-896.

[9] Cortes,C. and Vapnik, V. “Support-vector networks ”. Machine Learning, 1995, 20, pp.273-297. 